\title{
Perfil de personas con infección por VIH/SIDA: diferencial étnico, económico y socio-cultural en Chile
}

\author{
Ana M. Alarcón, Carolina Chahin, Sergio Muñoz, Marcelo Wolff y Rebeca Northland
}

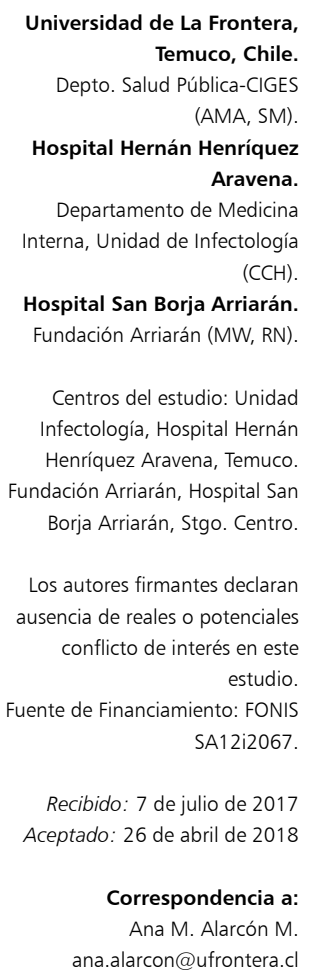

Centros del estudio: Unidad Infectología, Hospital Hernán Henríquez Aravena, Temuco. Fundación Arriarán, Hospital San Borja Arriarán, Stgo. Centro.

Los autores firmantes declaran ausencia de reales o potenciales conflicto de interés en este estudio.

Fuente de Financiamiento: FONIS SA12i2067.

Recibido: 7 de julio de 2017 Aceptado: 26 de abril de 2018

Correspondencia a: Ana M. Alarcón M. ana.alarcon@ufrontera.cl

\section{Introducción}

$\mathrm{D}$ esde la inclusión de la HAART (highly active antiretroviral therapy), la cifra de 40 millones de personas infectadas con VIH a nivel mundial ha bajado en $12 \%{ }^{1}$. Este descenso ha sido liderado por Estados Unidos de América y algunos países de Europa Occidental ${ }^{2,3}$. Sin embargo, de los 15 millones de personas con infección por VIH/SIDA en países de medianos y bajos ingresos, sólo 5,2 millones tienen acceso a tratamiento, siendo aún la cuarta causa de muerte más frecuente 4,5 .

En Chile, los casos de pacientes infectados por VIH han aumentado en el último quinquenio, existiendo anualmente 28,9 casos por cada 100.000 habitantes $\geq 13$ años y 500 muertes anuales. De los casos reportados por el Instituto de Salud Pública en Chile, 54,8\% viven en etapa de infección por VIH y 48,2\% están en etapa de SIDA activo. En nuestro país se infectan aproximadamente 5.000 casos anuales, con una prevalencia de $0,5 \%$ en el grupo de 13 a 49 años, y 65.000 casos anuales. Dentro de este rango, los jóvenes de 25 a 29 años, alcanzan las tasas más altas de infección con 50,6 por 100.000 habitantes ${ }^{6,7}$. La mortalidad se concentra en hombres (87\%) y en población económicamente activa (30 a 59 años). En las mujeres ha aumentado la notificación en etapa de infección por VIH. La principal vía de exposición al virus es la sexual ( $97 \%$ de los casos) siendo la relación entre hombres la más frecuente, pero con aumento de la vía de exposición heterosexual ${ }^{6}$.

La UNAIDS propone que existiendo baja prevalencia de VIH/SIDA, la prevención debería focalizarse en poblaciones claves, tales como, usuarios de drogas inyectables, comercio sexual, sexo entre hombres y migrantes ${ }^{5,8-11}$. El último informe sobre infección por VIH y poblaciones indígenas de la $\mathrm{OPS}^{12}$, incorpora el concepto de población emergente en infección por VIH/SIDA para referirse a la población indígena de América Latina. Hasta el momento, en nuestro país no existen estudios de prevalencia de infección por VIH/SIDA que desagreguen por etnicidad Mapuche ${ }^{13-15}$. Los datos provenientes de la Región Araucanía señalan que los pacientes Mapuche son pesquisados en salas UCI o en Unidades de Emergencia en condiciones extremadamente deterioradas y se asiste a un aumento significativo de casos de esta patología en esta población en los últimos 10 años ${ }^{16,17}$.

Existe una compleja interacción entre etnicidad y la patología del VIH/SIDA ${ }^{18-21}$. Por ejemplo: la presencia de nociones de corporalidad y sexualidad que entrañan relaciones de género y sexuales particulares; mecanismos de regulación de relaciones sexuales basados en parentesco, linaje y poder; sistemas de atención de salud tradicional que interactúan o colisionan con los biomédicos, y procesos migratorios que ubican a los indígenas en sectores 
urbanos con alto riesgo de contraer la infección por VIH/ SIDA $^{22-25}$.

Organismos internacionales como, OPS, OMS y UNAIDS, reconocen la existencia de factores sociales y culturales en el desarrollo y control del VIH/SIDA que se articulan con variables políticas y administrativas de salud de los países. En ese contexto, el modelo de Determinación Social de la Salud propuesto por la OPS, es una herramienta analítica que facilita la comprensión de los factores sociales, culturales y políticos en los resultados de salud $^{26}$. La etnicidad y situación socio-económica son, según este modelo, determinantes sociales que afectarían el acceso a tratamiento y pesquisa oportuna del VIH/SIDA.

La UNAIDS plantea que los mayores desafíos para el cumplimiento de la meta "Acceso Universal a la Prevención del VIH, Tratamiento, Cuidado y Apoyo para el año 2020"; será conocer las estructuras sociales, creencias y valores sociales que obstaculizan los esfuerzos de prevención de la infección por VIH; y abordar temas de pobreza, inequidad de género, de salud, etnia, y cualquier discriminación sobre los grupos vulnerables que impidan un mejor acceso a los beneficios del sistema social y de salud ${ }^{27}$.

\section{Objetivo}

Describir el perfil de los pacientes con infección por VIH/SIDA atendidos en dos centros de atención especializada de las regiones Araucanía y Metropolitana, de acuerdo a etnia Mapuche y no Mapuche, considerando tres grupos de variables:

- condiciones clínicas de los pacientes

- aspectos socio-económicos, y

- oportunidad terapéutica como estilo de vida y conducta sexual.

El propósito es contribuir con información válida, confiable y relevante para mejorar una oportunidad terapéutica a través de procesos de prevención y pesquisa temprana, entre poblaciones consideradas vulnerables o emergentes.

\section{Material y Método}

Estudio descriptivo, de corte transversal. La población de estudio estuvo compuesta por la totalidad de los pacientes activos del centro de la Región Araucanía (290 pacientes) y por una muestra no aleatoria consecutiva de 268 pacientes del centro de la Región Metropolitana. Los pacientes fueron seleccionados de acuerdo a los siguientes criterios: registro en la ficha clínica de pertenencia étnica Mapuche, nacionalidad chilena, mayor de 18 años y voluntad de participar en el estudio. La condición de etnicidad Mapuche fue triangulada con auto-identificación del paciente y presencia de uno o dos apellidos Mapuche ${ }^{28}$.
Los pacientes fueron reclutados por médicos o enfermeras de cada centro al momento de sus controles de salud, lo que se realizó hasta lograr el tamaño muestral establecido. El ingreso al estudio fue bajo consentimiento informado del paciente el que se separó del formulario de recolección de datos para resguardar su total confidencialidad, ello porque el consentimiento señala nombre y firma del paciente. El estudio contó con la aprobación de los Comités de Ética del Servicio de Salud Metropolitano Central y del Servicio de Salud Araucanía Sur.

La recolección de datos se realizó a través de un formulario que contenía tres secciones: la primera, datos clínicos provenientes de las fichas, la segunda, datos socioculturales y la tercera, datos sobre oportunidad terapéutica y diagnóstica; estas dos últimas secciones eran preguntas estructuradas que se formulaban directamente a los pacientes. En cada centro el equipo médico ingresó los datos clínicos de los pacientes al formulario de recolección de datos y posteriormente, un antropólogo en una sala privada de mismo centro, preguntaba por las variables socio-culturales y de oportunidad terapéutica. Las preguntas del formulario fueron diseñadas por el equipo de investigación basada en los objetivos del proyecto. Éstas fueron concretas y estructuradas, por ejemplo: ¿En cuál de los siguientes niveles de ingreso se ubica usted?, ¿Cuántas parejas sexuales ha tenido el último mes?, etc.

Las variables socio-culturales como, por ejemplo, el nivel de ingresos económicos se dividió en cuatro categorías (de $\leq 160.000$ a $>500.000$ pesos) ${ }^{* 29}$, y educación según último año cursado en tres categorías (de $\leq 8$ a $>12$ años). Las variables asociadas a oportunidad terapéutica y diagnóstica fueron: estilo de vida y conducta sexual; percepción de red de apoyo, motivo de examen, patologías asociadas a VIH/SIDA, y valores basales de LT CD4 al ingresar al programa. Se consideró pacientes con diagnóstico de SIDA aquellos con un recuento de LT CD4 $<200$ céls $/ \mathrm{mm}^{3}$, y aquellos con eventos definitorios de SIDA. Los datos se ingresaron al programa estadístico Stata. $14^{30}$ en el que se realizó análisis descriptivo de todas las variables, se evaluaron asociaciones crudas mediante pruebas de $\chi^{2}$ y estratificadas según Mantel-Haensen por cada variable en estudio, en especial Mapuche/no Mapuche que es la variable explicativa principal; y análisis de regresión logística múltiple para estimar una medida de magnitud y dirección de la asociación entre etnia y oportunidad diagnóstica y terapéutica controlando por el resto de las variables.

\section{Resultados}

Se describen a continuación los hallazgos en función de: caracterización socio-cultural y demográfica de los

*1.000 pesos $=1,6$ dólares americanos aproximadamente. 


\begin{tabular}{|c|c|c|c|}
\hline Variable & $\begin{array}{c}\text { Mapuche } \\
81\end{array}$ & $\begin{array}{c}\text { No-Mapuche } \\
477\end{array}$ & $p$ value \\
\hline \multicolumn{4}{|l|}{ Sexo } \\
\hline Femenino & 24 & 81 & 0,00 \\
\hline Masculino & 57 & 396 & \\
\hline Edad promedio (años) & 36,7 & 39,5 & 0,01 \\
\hline \multicolumn{4}{|l|}{ Ingresos en pesos* } \\
\hline$\leq 160.000$ & 55 & 44,5 & 0,12 \\
\hline $160-300.000$ & 16,3 & 22,4 & \\
\hline $300-500.000$ & 23,7 & 21,6 & \\
\hline$>500.000$ & 5,0 & 11,5 & \\
\hline \multicolumn{4}{|l|}{ Nivel educacional } \\
\hline$\leq 8$ años & 27,1 & 19,7 & 0,01 \\
\hline 9-12 años & 42 & 42,3 & \\
\hline$>12$ años & 30,9 & 38 & \\
\hline \multicolumn{4}{|l|}{ Actividad principal } \\
\hline Estudiante & 9,9 & 5,9 & 0,01 \\
\hline Dueña de casa & 21,0 & 9,2 & \\
\hline Trabajador no calificado & 35,8 & 36,9 & \\
\hline Servicios & 17,3 & 28,9 & \\
\hline Técnico-profesional & 16,1 & 18,2 & \\
\hline Comercio sexual & 0,0 & 0,1 & \\
\hline \multicolumn{4}{|l|}{ Orientación sexual } \\
\hline Heterosexual & 53,1 & 38 & 0,05 \\
\hline Homosexual & 43,2 & 53,5 & \\
\hline Bisexual & 3,7 & 8,5 & \\
\hline
\end{tabular}

pacientes del estudio, condiciones clínicas y factores asociados a oportunidad diagnóstica y terapéutica.

La Tabla 1 describe las características socio-demográficas de los sujetos de estudio diferenciados por etnia.

En las variables denominadas estilo de vida asociada a la infección por VIH/SIDA, se observan diferencias de acuerdo a etnia, ingresos y educación. La Tabla 2 muestra un mayor consumo de drogas, comercio sexual, sexo entre hombres y mayor cantidad de parejas sexuales en el último mes, reportado por pacientes no Mapuche. Excepto para comercio sexual, las diferencias en general se conservan según nivel de ingreso y educación. Llama además la atención que alrededor de $25 \%$ de los pacientes no utiliza preservativo.

En relación a la red de apoyo de los pacientes, la Tabla 3 muestra si la familia más cercana o personas con quienes vive saben de su enfermedad y si perciben apoyo de éstas. Los datos indican que no hay diferencias sobre la percepción de red apoyo por etnia, ingresos o educación. Poco más de la mitad de los pacientes reconocen sentirse apoyado por sus personas más cercanas.

El motivo de examen puede ser considerado una medida indirecta de la autopercepción de riesgo frente al VIH/SIDA. La Tabla 4 muestra que sólo el nivel educacional y de ingresos se asocia a esta autopercepción: estos pacientes se tomaron el examen por iniciativa propia o sospechar la posibilidad de contraer el VIH.

Aparte de explorar el perfil socio cultural y demográfico de los pacientes en control, un aspecto central del estudio fue explorar la asociación entre etnia, nivel de ingreso y educación, con algunas variables clínicas relevantes tales como co-morbilidad de depresión e ITS y la condición de

\begin{tabular}{|c|c|c|c|c|c|c|c|c|c|c|c|}
\hline & \multicolumn{2}{|c|}{ Alcohol } & \multicolumn{2}{|c|}{ Drogas } & \multicolumn{2}{|c|}{ Comercio sexual } & \multicolumn{2}{|c|}{ Uso de preservativo } & \multicolumn{2}{|c|}{ Sexo entre hombres } & \multirow{2}{*}{$\begin{array}{c}\text { n parejas sexuales/mes } \\
0 / \text { Una/ Dos o }+\end{array}$} \\
\hline & Sí & No & Sí & No & Sí & No & Sí & No & Sí & No & \\
\hline Etnia & \multicolumn{2}{|c|}{$(p=0,12)$} & \multicolumn{2}{|c|}{$(p=0,05)$} & \multicolumn{2}{|c|}{$(p=0,04)$} & \multicolumn{2}{|c|}{$(p=0,22)$} & \multicolumn{2}{|c|}{$(p=0,00)$} & $(p=0,01)$ \\
\hline Mapuche & 34,6 & 65,4 & 8,6 & 91,4 & 1,2 & 98,8 & 79 & 21 & 45,7 & 54,3 & $23,5 / 67,9 / 8,6$ \\
\hline No-Mapuche & 43,8 & 56,2 & 17,2 & 93,1 & 6,9 & 93,1 & 72,5 & 27,5 & 65,2 & 34,8 & 31 / 49,7 / 19,3 \\
\hline Ingresos en pesos* & \multicolumn{2}{|c|}{$(p=0,26)$} & \multicolumn{2}{|c|}{$(p=0,00)$} & \multicolumn{2}{|c|}{$(p=0,11)$} & \multicolumn{2}{|c|}{$(p=0,97)$} & \multicolumn{2}{|c|}{$(p=0,00)$} & $(p=0,00)$ \\
\hline$\leq 160.000$ & 39,4 & 60,6 & 10,6 & 89,4 & 8,6 & 91,4 & 73,1 & 26,9 & 43,7 & 56,3 & $35,9 / 54,3 / 9,8$ \\
\hline $160-300.000$ & 49,2 & 50,8 & 13,3 & 86,7 & 4,2 & 95,8 & 72,5 & 27,5 & 74,2 & 25,8 & $27,5 / 52,5 / 20$ \\
\hline $300-500.000$ & 44,3 & 55,7 & 26,2 & 73,8 & 4,9 & 95,1 & 74,6 & 25,4 & 80,3 & 19,7 & $27,1 / 46,7 / 26,2$ \\
\hline$>500.000$ & 37,3 & 62,7 & 23,7 & 76,3 & 1,7 & 98,3 & 73,4 & 26,6 & 83,0 & 17,0 & $15,3 / 54,2 / 30,5$ \\
\hline Nivel educacional & \multicolumn{2}{|c|}{$(p=0,00)$} & \multicolumn{2}{|c|}{$(p=0,00)$} & \multicolumn{2}{|c|}{$(p=0,10)$} & \multicolumn{2}{|c|}{$(p=0,02)$} & \multicolumn{2}{|c|}{$(p=0,00)$} & $(p=0,00)$ \\
\hline$\leq 8$ años & 38,8 & 61,2 & 11,2 & 88,8 & 8,7 & 91,3 & 75,9 & 24,1 & 30,2 & 69,8 & $26,7 / 62,1 / 11,2$ \\
\hline 9-12 años & 37 & 63 & 10,3 & 89,7 & 6,2 & 93,8 & 73,2 & 26,8 & 55,8 & 44,2 & 34,2 / 38,2 / 26,7 \\
\hline$>12$ años & 50 & 50 & 24,3 & 75,7 & 4,5 & 95,4 & 72,5 & 27,5 & 86,2 & 13,8 & $25,2 / 47,7 / 7,1$ \\
\hline
\end{tabular}




\begin{tabular}{|c|c|c|c|c|c|c|}
\hline & \multicolumn{2}{|c|}{$\begin{array}{l}\text { Familia más cercana sabe } \\
\text { de su enfermedad }\end{array}$} & \multicolumn{2}{|c|}{$\begin{array}{l}\text { Personas con quien vive saben } \\
\text { de su enfermedad }\end{array}$} & \multicolumn{2}{|c|}{$\begin{array}{l}\text { Siente apoyo de las personas cor } \\
\text { quien vive o sus más cercanos }\end{array}$} \\
\hline & Sí & No & Sí & No & Sí & No \\
\hline Etnia & \multicolumn{2}{|c|}{$(p=0,42)$} & \multicolumn{2}{|c|}{$(p=0,51)$} & \multicolumn{2}{|c|}{$(p=0,50)$} \\
\hline Mapuche & 53,1 & 46,9 & 65,4 & 34,6 & 55,6 & 44,4 \\
\hline No Mapuche & 57,9 & 42,1 & 61,6 & 38,4 & 59,5 & 40,5 \\
\hline Ingresos en pesos* & \multicolumn{2}{|c|}{$(p=0,10)$} & \multicolumn{2}{|c|}{$(p=0,20)$} & \multicolumn{2}{|c|}{$(p=0,91)$} \\
\hline$\leq 160.000$ & 59,8 & 40,2 & 66,4 & 55,6 & 59,4 & 40,6 \\
\hline $160-300.000$ & 58,3 & 41,7 & 61,7 & 38,3 & 60,8 & 39,2 \\
\hline $300-500.000$ & 47,5 & 52,5 & 56,6 & 43,4 & 57,4 & 42,6 \\
\hline$>500.000$ & 62,7 & 37,3 & 55,9 & 44,1 & 55,9 & 44,1 \\
\hline Nivel educacional & \multicolumn{2}{|c|}{$(p=0,35)$} & \multicolumn{2}{|c|}{$(p=0,03)$} & \multicolumn{2}{|c|}{$(p=0,31)$} \\
\hline$\leq 8$ años & 62,9 & 37,1 & 69,8 & 30,2 & 62,1 & 37,9 \\
\hline 9-12 años & 55,1 & 44,9 & 64 & 36 & 61 & 38,9 \\
\hline$>12$ años & 56,3 & 43,7 & 55,8 & 44,2 & 54,9 & 45,1 \\
\hline
\end{tabular}

\begin{tabular}{|c|c|c|c|c|c|c|}
\hline & Iniciativa propia & Control embarazo & Contacto personas con VIH & Sospecha enfermedad & Otros & $p$ value \\
\hline \multicolumn{7}{|l|}{ Etnia } \\
\hline Mapuche & 19,8 & 4,9 & 17,3 & 24,7 & 33,3 & 0,90 \\
\hline No Mapuche & 20,9 & 3,2 & 20,2 & 23,2 & 32,6 & \\
\hline \multicolumn{7}{|c|}{ Nivel educacional } \\
\hline$\leq 8$ años & 13,8 & 6,9 & 15,5 & 19,8 & 44 & 0,00 \\
\hline 9-12 años & 19,5 & 4,7 & 21,6 & 22,0 & 32,2 & \\
\hline$>12$ años & 26,1 & 0 & 20,1 & 27,1 & 26,3 & \\
\hline \multicolumn{7}{|c|}{ Ingresos en pesos } \\
\hline$\leq 160.000$ & 17,7 & 6,3 & 14,5 & 24,3 & 37,3 & \\
\hline $160-300.000$ & 21,2 & 1,7 & 23,7 & 20,3 & 33,0 & 0,00 \\
\hline $300-500.000$ & 23,1 & 0,8 & 26,5 & 22,3 & 27,3 & \\
\hline$>500.000$ & 28,6 & 0 & 21,4 & 26,8 & 23,2 & \\
\hline
\end{tabular}

ingreso a tratamiento de los pacientes según recuento de LT CD4. En patologías como depresión e infecciones de transmisión sexual, la Tabla 5 muestra que sólo el nivel de ingreso económico del paciente se asocia a la presencia de menor depresión (diagnosticada por psiquiatra).

Según la etapa de enfermedad (VIH o SIDA), en que ingresan los pacientes a tratamiento, se observa que aquellos con menor nivel educacional y menor ingreso se encuentran en etapa SIDA es decir con un recuento de LT CD4 $<200$ céls $/ \mathrm{mm}^{3}$. En este aspecto la etnia del paciente no fue relevante. La Tabla 6 indica estos hallazgos.

Respecto del recuento basal de LT CD4 (Tabla 7), se observa que la mediana de ingreso de los pacientes Mapuche al centro de atención de VIH/SIDA es con un valor de LT CD4 $\leq 51$ céls $/ \mathrm{mm}^{3}$. Éstos se encuentran bajo el percentil 25 (IC 38-123) versus los no Mapuche, en que $25 \%$ tiene LT CD4 76 céls $/ \mathrm{mm}^{3}$ (IC 55-99). Asimismo,
Tabla 5. Patologías asociadas (Infecciones de Transmisión Sexual y Depresión) según etnia, nivel educacional e ingreso

\begin{tabular}{|c|c|c|c|c|c|c|}
\hline & \multicolumn{2}{|l|}{ ITS } & $p$ value & \multicolumn{2}{|c|}{ Depresión } & p value \\
\hline \multicolumn{7}{|l|}{ Etnia } \\
\hline Mapuche & 39,5 & 60,5 & 0.62 & 204 & 79.6 & 0.69 \\
\hline No Mapuche & 36,7 & 63,3 & & 18,5 & 81,5 & \\
\hline \multicolumn{7}{|c|}{ Nivel educacional } \\
\hline$\leq 8$ años & 30,2 & 69,8 & 0,19 & 25 & 75 & 0,06 \\
\hline 9-12 años & 37,7 & 62,3 & & 22,1 & 77,9 & \\
\hline$>12$ años & 40,3 & 59,7 & & 15,1 & 84,9 & \\
\hline \multicolumn{7}{|c|}{ Ingresos en pesos } \\
\hline$\leq 160.000$ & 32 & 68 & 0,16 & 25,8 & 74,2 & \\
\hline $160-300.000$ & 42,5 & 57,5 & & 17,5 & 82,5 & 0,01 \\
\hline $300-500.000$ & 40,2 & 59,8 & & 15,7 & 84,3 & \\
\hline$>500.000$ & 40,7 & 59,3 & & 20,2 & 79,8 & \\
\hline
\end{tabular}




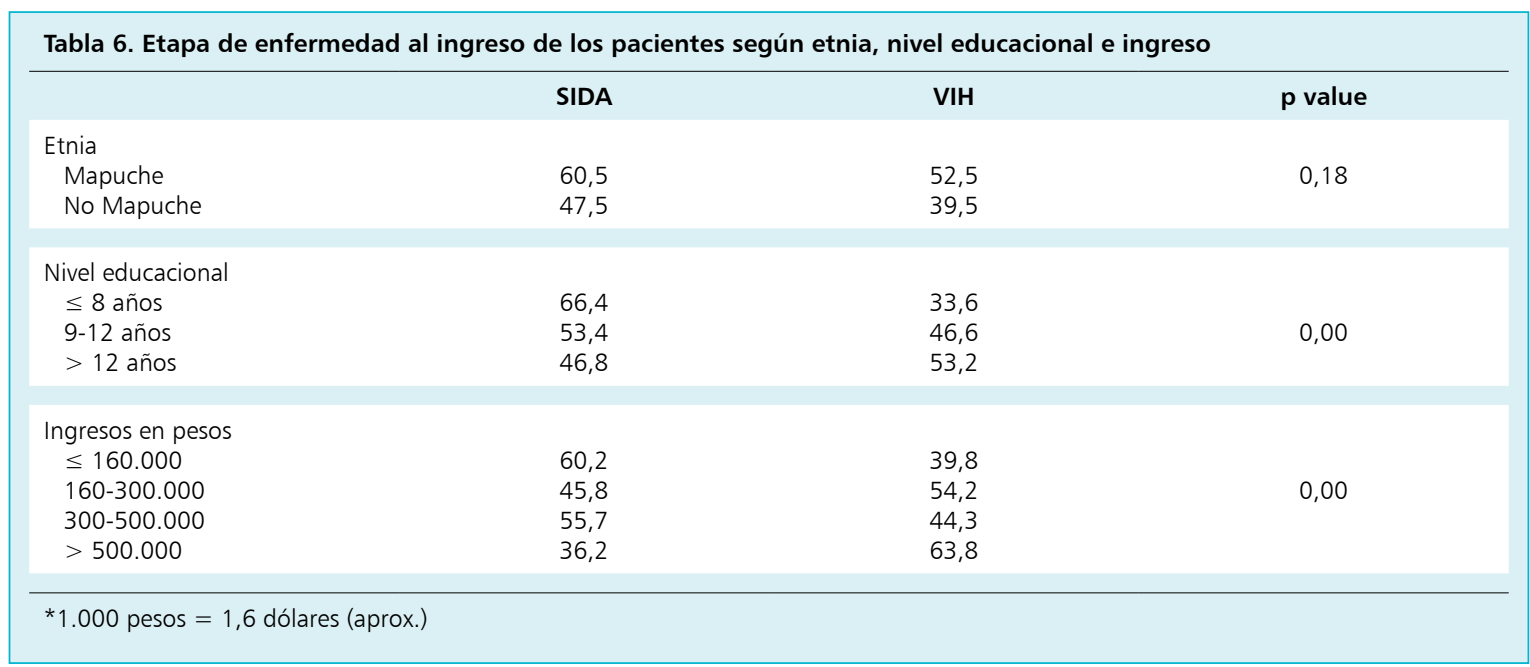

\begin{tabular}{|c|c|c|c|}
\hline & Percentil 25 (IC) & Percentil 50 (IC) & Percentil 75 (IC) \\
\hline \multicolumn{4}{|l|}{ Etnia: } \\
\hline Mapuche & $51,5(38-123)$ & $226(147,1-281,6)$ & $417,5(315,3-547)$ \\
\hline No Mapuche & $76,5(55-99,1)$ & $233(203-274,8)$ & $417(383-453)$ \\
\hline \multicolumn{4}{|l|}{ Nivel educacional } \\
\hline$\leq 8$ años & $38(29,5-65,6)$ & $161(96,8-208,8)$ & $319(277,3-389,1)$ \\
\hline 9-12 años & $104(865,3-128,7)$ & $252(208.8-289,8)$ & $453,5(383,2-504,8)$ \\
\hline$>12$ años & $75,5(48,1-117.8)$ & $270(206-314)$ & $432,5(402,5-470,7)$ \\
\hline \multicolumn{4}{|l|}{ Ingresos en pesos } \\
\hline$\leq 160.000$ & $48,2(34-70,2)$ & $200(166-247)$ & $378,7(324-436)$ \\
\hline $160-300.000$ & $104(59-153)$ & $264,5(215,5-342)$ & $469,2(378-553)$ \\
\hline $300-500.000$ & $86,8(55-130,4)$ & $240,5(169-311,3)$ & $423((373,9-509,1)$ \\
\hline$>500.000$ & $136(55,1-206)$ & $303(220,4-396,5)$ & $503(404,2-610,9)$ \\
\hline
\end{tabular}

quienes tienen menos educación e ingresos presentan recuentos de LT CD4 más bajos al iniciar tratamiento, es decir acuden en etapas más avanzadas del proceso de enfermedad.

Finalmente, se realizó un análisis para comparar la etapa de enfermedad al ingreso a tratamiento y la presencia de pacientes Mapuche y no Mapuche según centro de estudio. Este análisis indicó que 58,3\% de los pacientes en Araucanía ingresan en etapa SIDA, y que de éstos la mayoría son Mapuche.

\section{Discusión}

El perfil general descrito en los pacientes de este estudio comparte la mayoría de las características reportadas en nuestro país y otros de Latinoamérica ${ }^{31}$. Es una patología fundamentalmente de la población masculina, de hombres que tienen sexo con hombres, en edad productiva, cuya actividad principal es ser estudiante, trabajar en servicios o trabajos no calificados, con bajos niveles de ingresos y educación menor a 12 años. Sin embargo, al desagregar por etnia Mapuche, este perfil cambia hacia una población mayormente heterosexual, un poco más joven, de menores niveles de ingresos y educación que los pacientes no Mapuche. Son además en su mayoría trabajadores no calificados y hay una importante proporción de mujeres dueñas de casa.

La mayoría de los pacientes del estudio llegaron al centro de atención en etapa SIDA - considerada ingreso tardío de la patología-, por ende, mayor riesgo de morbilidad y mortalidad. La mediana de ingreso de LT CD4 a un programa de control y tratamiento de VIH/SIDA en Chile es de 233 céls $/ \mathrm{mm}^{36}$; en este estudio se encontró que la mediana fue menor a 100 céls $/ \mathrm{mm}^{3}$, y que los pacientes 
Mapuche tuvieron los recuentos de LT CD4 más bajos de la muestra.

Los pacientes con mayor nivel de educación e ingresos y que coinciden con etnia no Mapuche, tienden a realizarse el examen por iniciativa propia o sospecha de contraer la enfermedad. Además, la mayoría de ellos ingresa a control en etapa de infección por VIH, lo cual le confiere un mejor pronóstico en comparación con aquellos pacientes de baja escolaridad e ingresos.

Llama la atención en la mayoría de los pacientes, su percepción de poco apoyo de familiares cercanos, o de las personas con quien vive. Aunque esta cifra no es estadísticamente significativa, parece relevante desde una perspectiva social y clínica, puesto que tener una buena red de apoyo influye notablemente en el éxito terapéutico ${ }^{32,33}$.

El análisis por centro de atención y etnia Mapuche mostró que los pacientes Mapuche de la Región Araucanía ingresaron en etapas más tardías de enfermedad; incluso, estos pacientes mostraron niveles de LT CD4 porcentualmente más bajos que los Mapuche de la Región Metropolitana.

Es importante enfatizar que la condición de vulnerabilidad para efectos del VIH/SIDA está en la población indígena Mapuche, con baja educación y bajos niveles socio-económicos. Estas condiciones sociales continúan siendo barreras que afectan la equidad en las oportunidades en salud ${ }^{34}$.

En este contexto, se debería comenzar una era de enfoque socio-cultural de la enfermedad, donde se aborden los determinantes sociales de la salud ${ }^{35}$. Este estudio demuestra la existencia de una brecha social vinculada a tratamiento y pronóstico de la infección por VIH/SIDA; es decir personas con mayor educación e ingresos tienen mejor pronóstico pues en su mayoría llegan en etapa de infección por VIH; a su vez en este grupo, quienes tienen menor posibilidad de recuperación son los Mapuche (por su nivel de LT CD4) y de éstos los que viven en Araucanía.

Chile, al declararse país pluriétnico que acoge al Convenio 169, debe reconocer y abordar las diferencias étnico-culturales en cada una de sus políticas públicas. La variable etnicidad Mapuche es un factor que hace diferencia en nuestro país en ámbitos de salud-enfermedad y, como se indicó en este estudio, la pertenencia étnica Mapuche, intervino en el pronóstico de la infección por VIH/SIDA para profundizar brechas en salud. En este contexto, la etnicidad Mapuche constituye una determi- nante social de enfermedad. Ello significa que nuestro país debería asumir el compromiso de profundizar en el conocimiento sobre etnia Mapuche y VIH/SIDA y buscar mecanismos de prevención y promoción adecuados a las percepciones de enfermedad de los pacientes étnicamente diferenciados.

Respecto de las limitaciones del estudio, se podría apuntar a que las inferencias representan a una pequeña porción de la población de pacientes que se encuentra en control y tratamiento en el país. Asimismo, se enfrentó un importante desafío en el proceso de investigación para acceder a personas con infección por VIH/SIDA, lo que fue particularmente complejo entre los pacientes Mapuche. Una de las fortalezas de este estudio ha sido el abordaje interdisciplinario lo cual permitió dar cuenta de temas culturales que se abordarán en estudios futuros.

\section{Resumen}

Introducción: La asociación entre etnicidad e infección por VIH/SIDA constituye un tema emergente y poco explorado en Chile. Objetivo: Describir el perfil de pacientes con infección por VIH/SIDA según etnia Mapuche y no Mapuche asociados a condiciones clínicas, factores socio-económicos y oportunidad terapéutica en pacientes de dos centros de atención de las regiones Araucanía y Metropolitana, Chile. Material y Método: Estudio de corte transversal con 558 pacientes cuyos datos se recolectaron mediante un formulario que contenía las variables del estudio, obtenidos tras consentimiento informado. Se realizó análisis descriptivo, asociaciones crudas y estratificadas por cada variable. Resultados: Los pacientes Mapuche fueron en su mayoría de sexo masculino, $53,1 \%$ heterosexuales, edad promedio 36,7 años, y nivel educacional e ingresos más bajos que los no Mapuche. La mediana de LT CD4 basal fue más baja en pacientes Mapuche, 51 céls $/ \mathrm{mm}^{3}$ o menos y bajo el percentil 25 (IC 38-123). Se observó que existe mayor consumo de drogas, comercio sexual, sexo entre hombres y mayor número de parejas sexuales en último mes, en personas de la etnia no Mapuche y que, además, tienen mejores niveles de ingreso y educación. Conclusión: Existen perfiles diferenciados según etnia respecto de la condición sociocultural y de ingreso de los pacientes a control, generando inequidad en un tratamiento oportuno hacia los pacientes Mapuche, de baja escolaridad y menor educación.

\section{Referencias bibliográficas}

1.- UNAIDS Report on the Global AIDS epidemic 2013. Joint United Nations Programme on HIV/ AIDS (UNAIDS). World Health Organization Library Cataloguing-in-Publication Data.
Disponible en: http://www.unaids.org/sites/ default/files/media_asset/UNAIDS_Global_ Report_2013_en_1.pdf(Acceso en marzo de 2016).

2.- Survival after introduction of HAART in people with known duration of HIV-1 infection. The
CASCADE Collaboration. Concerted Action on SeroConversion to AIDS and Death in Europe. Lancet 2000; 355: 1158-9. DOI: https://doi. org/10.1016/S0140-6736(00)02069-9.

3.- Alonso M, Martin L, Muñoz S, Jacobson J. Patterns, trends and sex differences in HIV/ 
AIDS reported mortality in Latin American countries: 1996-2007. BMC Public Health 2011; 11: 605-16. doi: 10.1186/1471-2458-11605

4.- World Health Organization. Global health risks: Mortality and burden of disease attributable to selected major risks. Geneva: World Health Organization; 2009 (Acceso en abril de 2016).

5.- UNAIDS Data 2017. Joint United Nations Programme on HIV/AIDS (UNAIDS). World Health Organization Library Cataloguingin-Publication Data. Disponible en: http:// www.unaids.org/sites/default/files/media asset/20170720_Data_book_2017_en.pdf. (Acceso en septiembre de 2017).

6.- Ministerio de Salud República de Chile, Unidad de Epidemiología. Informe Epidemiológico Preliminar de las Infecciones de Transmisión Sexual en Chile hasta el año 2017. Documento Interno.

7.- Instituto de Salud Pública. Boletín de Vigilancia de Laboratorio. Resultados confirmación de infección por VIH en Chile 2010-2015. http:// www.ispch.cl/sites/default/files/BoletinVIH27022017B.pdf. (Acceso en abril de 2017).

8.- Tuboi S H, Schechter M, McGowan C C, Cesar C, Krolewiecki A, Cahn P, et al. Mortality during the first year of potent antiretroviral therapy in HIV-1-infected patients in 7 sites throughout Latin America and the Caribbean. J Acquir Immune Defic Syndr 2009; 51: 615-23. doi: 10.1097/QAI.0b013e3181a44f0a.

9.- The ART-LINC Collaboration of the International Databases to Evaluate AIDS (IeDEA). Antiretroviral therapy in resourcelimited settings 1996 to 2006: patient characteristics, treatment regimens and monitoring in sub-Saharan Africa, Asia and Latin America. Trop Med Int Health 2008; 13: 870-9. doi: 10.1111/j.13653156.2008.02078.x.

10.- Carael M, Marais H, Polsky J, y Mendoza A Is there a gender gap in the HIV response? Evaluating national HIV responses from the United Nations General Assembly Special Session on HIV/AIDS country reports. J Acquir Immune Defic Syndr 2009; 52: S111-S118. doi: 10.1097/ QAI.0b013e3181baeec2.

11.- World Health Organization. Monitoring equity in access to AIDS treatment programmes: a review of concepts, models, methods and indicators Library Cataloguing-in-Publication Data. 2010. (Acceso en septiembre de 2017).

12.- Panamerican Health Organization. Promotion of healthy sexual behavior and prevention of HIV-AIDS among indigenous people in America. Abya Yala. Quito 2003, p 207.

13.- Manríquez J, Stuardo V. Adherencia a la TARV en VIH: la importancia del contexto sociocultural implicado. Rev Chilena Infectol 2015; 32: 361-2. http://dx.doi.org/10.4067/S0716-

\section{8.}

14.- Martínez P, Olea A, Chiu M. Situación epidemiológica de la infección por VIHSIDA en Chile. Rev Chilena Infectol 2006; 23: 321-9. http://dx.doi.org/10.4067/S071610182006000400005.

15.- Centro Latinoamericano y Caribeño de Demografía. División de Población de la CEPAL. Inmigración internacional en América Latina. 2011. Disponible en: http://repositorio. cepal.org/bitstream/handle/11362/35288/ S1100998_es.pdf. (Acceso en octubre de 2107).

16.- Ministerio de Salud de Chile. Departamento de Epidemiología. División de Planificación Sanitaria. Informe Nacional: evolución de la infección por VIH/SIDA Chile 1984-2012. Rev Chilena Infectol. 2015; 32: 17-43. http://dx.doi. org/10.4067/S0716-10182015000100003.

17.- Aukinko Zomo. Mapuche Corporation of Mapuche Women. Sexualidad y prevención del VIH SIDA entre adolescentes Mapuche. Aukinko, Temuco 2006, p 198.

18.- Ministerio de Salud Chile. En los dominios de la salud y la cultura: estudio de caracterización de los factores de riesgo y vulnerabilidad frente al VIH/SIDA en pueblos originarios. 2006. Disponible en https://www.researchgate.net/ publication/265675957_COORDINADORAS EN_LOS_DOMINIOS_DE_LA_SALUD_Y LA_CULTURA (Acceso en marzo de 2017).

19.- Buela-Casal G, Bermúdez M, Sánchez G, y De Los Santos- Roig M. Situación del VIH/SIDA en Latinoamérica al final del siglo XX: Análisis de las diferencias entre países. Rev Med Chile 2001; 129: 944-54. http://dx.doi.org/10.4067/ S0034-98872001000800015.

20.- Rhodes A, Merrill Singer B, Philippe B, Friedman S, Strathdee S. The social structural production of HIV risk among injecting drug users. Soc Sci Med 2005; 61:1026-44. DOI: 10.1016/j.socscimed.2004.12.024.

21.- Betancourt C, Pinilla M. Apreciaciones sobre el contexto sociocultural del VIH-Sida en las comunidades indígenas en Colombia. Desacatos. Revista de Antropología Social 2011; 35: 75-86. DOI: https://doi. org/10.29340/35.322.

22.- Promoción de la salud sexual y prevención del VIH-SIDA y de las ITS en los pueblos indígenas de las Américas. 2003. Unidad de VIH/SIDA e ITS Área de Salud de la Familia y Comunitaria, Organización Panamericana de la Salud. Abya-Yala Kuyarinakui Eds. p 207.

23.- Chow AL, Taylor T, Salgado J. La problemática del VIH-SIDA entre los pobladores del casco urbano de Laguna de Perlas, RAAS, 2007 -200. Ciencia e interculturalidad 2008; 3: 98 114. http://revistas.uraccan.edu.ni/index.php/ Interculturalidad/article/view/160.

24.- Farmer P. Chapter 2: Rethinking emerging infectious diseases. 5 th. Infections and
Inqualities: The modern plagues P. Farmer. Los Angeles: University California Press; 2000, p 37-59.

25.- Tenorio R. La intimidad desnuda. Sexualidad y cultura indígena. Quito: Abya-Yala; 2004, p 205. http://digitalrepository.unm.edu/cgi/ viewcontent.cgi? article $=1094 \&$ context $=$ abya yala.

26.- Modelo determinación social de la salud: En: http://www.who.int/social_determinants/es/ (Acceso en marzo de 2017).

27.- UNAIDS Report on the Global AIDS Epidemic 2010. Joint United Nations Programme on HIV/ AIDS (UNAIDS). World Health Organization Library Cataloguing-in-Publication Data. Disponible en http://www.unaids.org/en/ resources/documents/2010/20101123 globalreport (Acceso en diciembre de 2016).

28.- Instituto Nacional de Estadísticas 2005. Enfoque estadístico pueblos indígenas en Chile. Documento INE y Programa Orígenes Mideplan-BID. CONADI Disponible en http://www.ine.cl/canales/chile_estadistico/ estadisticas_sociales_culturales/etnias/pdf/ estadisticas_indigenas_2002_11_09_09.pdf. (Acceso en enero de 2016).

29.- Asociación Chilena de Empresas de Investigación de Mercado (2008). Grupos Socioeconómicos. Disponible en http://www.aimchile.cl/wp-content/ uploads/2011/12/Grupos_Socioeconomicos_ AIM-2008.pdf. (Acceso en enero de 2016).

30.- Stata.12 Data Analysis and Statistical Software.

31.- Gouws E, Cuchi P. International Collaboration on Estimating HIV Incidence by Modes of Transmission. Focusing the HIV response through estimating the major modes of HIV transmission: a multi-country analysis. Sex Transm Infect 2012; 88 (Suppl 2): i76-85. doi: 10.1136/sextrans-2012-050719.

32.- Cazenave G, Ferrer S, Castro B, y Cuevas A. El familiar cuidador de pacientes con SIDA y la toma de decisiones en salud. Rev Chilena Infectol 2005; 22: 51-7. http://dx.doi. org/10.4067/S0716-10182005000100007.

33.- Ballester Arnal R. Eficacia terapéutica de un programa de intervención grupal cognitivo compartamental para mejorar la adhesión al tratamiento y el estado emocional de pacientes con infección por VIH/SIDA. Psicothema 2005; 15: 517-23. http://www.psicothema.com/ pdf/1101.pdf.

34.- Ponce P, Núñez G. Pueblos indígenas y VIHSIDA Desacatos 2011; 35: 7-10. http://www. scielo.org.mx/pdf/desacatos/n35/n35a1.pdf

35.- García González R. El enfoque social como elemento esencial del quehacer por la salud de la población. Rev Cubana Salud Pública 2011; 37:675-85. http://scielo. sld.cu/scielo.php?script=sci_arttext\&pid $=$ S0864-34662011000500015. 